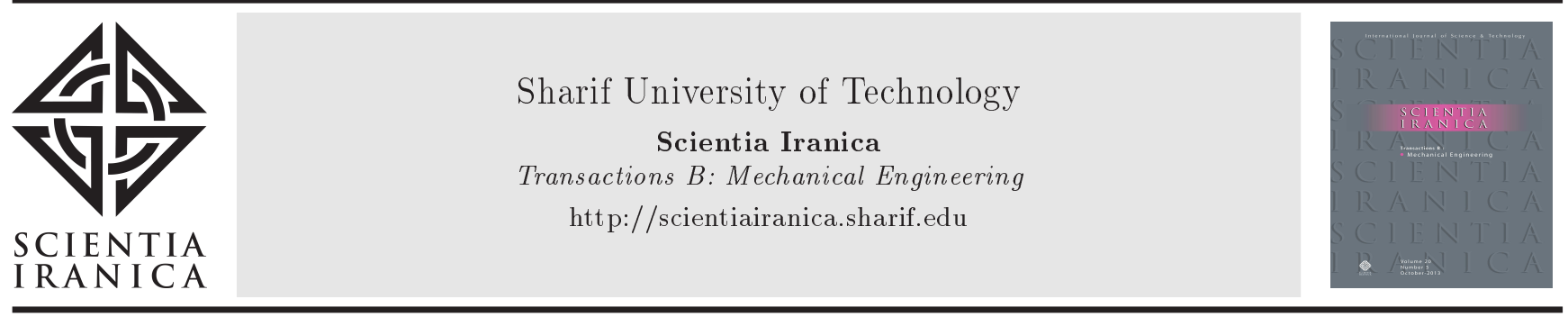

\title{
A combined approximation method for nonlinear foam drainage equation
}

\author{
M. Izadi* \\ Department of Applied Mathematics, Faculty of Mathematics and Computer, Shahid Bahonar University of Kerman, Kerman, Iran.
}

Received 8 August 2020; received in revised form 14 March 2021; accepted 24 May 2021

KEYWORDS
Foam drainage
equation;
Bessel functions;
Collocation points;
Nonlinear PDE;
Taylor series
expansion.

\section{KEYWORDS}

Bessel functions;

Collocation points

expansion.

\begin{abstract}
The aim of this study is to develop a combined approximation technique to find a numerical solution to the foam drainage equation in various absorption and distillation processes. In this approach, first, discretization of time is performed with the aid of the Taylor expansion series. Hence, a collocation method based on novel Bessel polynomials is utilized for the space variable. Thus, the solution is found by solving a linear system of algebraic equations in each time step. Numerical simulations are provided to check the accuracy and efficiency of the presented algorithm. The numerical results are compared with exact solutions as well as the outcomes of other existing numerical methods.
\end{abstract}

(C) 2022 Sharif University of Technology. All rights reserved.

\section{Introduction}

This research aims to develop an efficient approximation algorithm to solve the nonlinear foam drainage equation [1]:

$$
\frac{\partial W}{\partial t}+\frac{\partial}{\partial x}\left(W^{2}-\frac{\sqrt{W}}{2} \frac{\partial W}{\partial x}\right)=0
$$

with initial condition:

$$
\left.W\right|_{t=0}=W_{0}(x) .
$$

Here, $W(x, t)$ as the scaled coordinates and $t$ the time position) denotes the cross-section of a channel formed where three films meet, usually indicated as "Plateau border". Foams naturally appear in numerous applications and technological processes and have attracted the attention of many researchers given their significance, see $\mathrm{cf}$. $[2,3]$.

\footnotetext{
*.E-mail address: izadi@uk.ac.ir
}

By substituting $W(x, t):=w^{2}(x, t)$ in Eqs. (1) and (2), we arrive at the following nonlinear initialvalue problem:

$$
\left\{\begin{array}{r}
w_{t}+2 w^{2} w_{x}-w_{x}^{2},-\frac{1}{2} w w_{x x}=0, \\
0 \leq x \leq L, \quad 0 \leq t \leq T \\
\left.w\right|_{t=0}=w_{0}(x):=\sqrt{W_{0}(x)}, \quad 0<x<L
\end{array}\right.
$$

where $T>0$ is a given final time and $L>0$ is a real constant. In addition, the following boundary conditions are supplemented with the initial-value problem (3):

$$
w(0, t)=h_{0}(t), \quad w(L, t)=h_{1}(t), \quad 0 \leq t \leq T,
$$

where $h_{0}(t)$ and $h_{1}(t)$ are two prescribed functions. Over the last few decades, researchers have proposed several analytical techniques as well as approximative algorithms to solve the foam drainage equation. Among these methods, we mention the Tahn and Adomian decomposition methods [4], the Homotopy Perturbation Method (HPM) [5], the symmetry Lie group approaches [6,7], the series solution based on the homotopy analysis method [8], the Exp-function 
approach [9], the Homotopy Perturbation Transform Method (HPTM) [10], a semi-analytical approach based upon the quasilinearization and the Haar wavelet bases, and a hybrid computational approach based on the generalized Chebyshev polynomials and quasilinearization technique [11].

The main objective of this research study is to derive a new combined approximation technique based on a combination of Taylor-series approach and spectral collocation scheme for the numerical treatment of the nonlinear foam-drainage equation. On the one hand, the considered equation is a time-dependent model problem and, thus, it is of interest to develop an accurate time-marching algorithm for our model. On the other hand, collocation-based methods have been applied successfully to many model problems in science and engineering due to their efficiency as well as simple applicability while giving high accuracy. Among many existing approaches based on the collocation strategy, we can mention the meshfree methods $[12,13]$, the spectral collocation approach based on diverse polynomial bases such as Legendre, Chebyshev, Chelyshkov, etc. utilized in [14-17], over the past decades.

In approximate terms, the Taylor approach with second-order accuracy is first employed to discretize the time variable. Then, in each time step, it is supposed that the underlying model problem has a solution in terms of the novel Bessel series expansion of the unknown function. Afterwards, representing all involved unknowns in the Bessel matrix form together with the proper usage of a suitable set of collocation points helps determine the unknown series coefficients by solving a linear system of matrix equations. Indeed, the Bessel polynomial of order $\ell$ is defined explicitly as [18]:

$$
\mathbb{B}_{\ell}(x)=\sum_{\kappa=0}^{\ell} \frac{(\ell+\kappa) !}{(\ell-\kappa) !} \frac{x^{\kappa}}{2^{\kappa} \kappa !}, \quad \ell=0,1, \ldots .
$$

See also [19-22] for recent applications. Besides the fact that all coefficients of $\mathbb{B}_{\ell}(x)$ are positive, they also satisfy the second-order differential equation:

$$
x^{2} \mathbb{B}_{\ell}^{\prime \prime}(x)+2(x+1) \mathbb{B}_{\ell}^{\prime}(x)-\ell(\ell+1) \mathbb{B}_{\ell}(x)=0 .
$$

It should be noted that the considered Bessel functions $\mathbb{B}_{\ell}(x)$ differ from the traditional Bessel functions of the first kind, which have previously been utilized in various research papers, see cf. [17,23].

\section{Taylor scheme for time discretization}

First, an attempt is made to discretize the foam drainage equation with respect to time variable. In this respect, the interval $[0, T]$ can be subdivided into $(M+1)$ grid points:

$$
0=: t_{0}<t_{1}=\Delta t<\ldots<t_{M}:=M \Delta t=T,
$$

being $\Delta t=t_{n}-t_{n-1}$ the uniform time step. To get a time-accurate discretization scheme, according to the Taylor series representation for $w^{n}=w\left(x, t_{n}\right)$ we obtain the following:

$$
w_{t}^{n}=\frac{w^{n+1}-w^{n}}{\Delta t}-\frac{1}{2} \Delta t w_{t t}^{n}+\mathcal{O}\left(\Delta t^{2}\right) .
$$

To proceed, we differentiate Eq. (3) with respect to $t$ to get:

$$
\begin{aligned}
w_{t t}^{n}= & {\left[\frac{1}{2} w^{n} w_{x x}^{n}+\left(w_{x}^{n}\right)^{2}-2\left(w^{n}\right)^{2} w_{x}^{n}\right]_{t} } \\
= & \frac{1}{2} w_{t}^{n} w_{x x}^{n}+\frac{1}{2} w^{n}\left(w_{t}^{n}\right)_{x x}+2 w_{x}^{n}\left(w_{t}^{n}\right)_{x} \\
& -4 w^{n} w_{t}^{n} w_{x}^{n}-2\left(w^{n}\right)^{2}\left(w_{t}^{n}\right)_{x} .
\end{aligned}
$$

By replacing the first-order derivatives $w_{t}^{n} \approx\left(w^{n+1}-\right.$ $\left.w^{n}\right) / \Delta t$ in all occurrences, we may write $w_{t t}^{n}$ as follows:

$$
\begin{aligned}
\Delta t w_{t t}^{n}= & {\left[\frac{1}{2} w_{x x}^{n}-4 w^{n} w_{t}^{n}\right]\left(w^{n+1}-w^{n}\right) } \\
& +2\left[w_{x}^{n}-\left(w^{n}\right)^{2}\right]\left(w_{x}^{n+1}-w_{x}^{n}\right) \\
& +\frac{1}{2} w^{n}\left(w_{x x}^{n+1}-w_{x x}^{n}\right) .
\end{aligned}
$$

Next, Eq. (7) is inserted into the right-hand side of Eq. (6) using the time discretized form of Eq. (3), i.e.:

$$
w_{t}^{n}=-2\left(w^{n}\right)^{2} w_{x}^{n}+\left(w_{x}^{n}\right)^{2}+\frac{1}{2} w^{n} w_{x x}^{n},
$$

for the left-hand side of Eq. (6). After some manipulations, the following time discretized equation for Eq. (3) with second-order accuracy in time is obtained:

$$
\begin{aligned}
& {\left[\Delta t\left(2 w^{n} w_{x}^{n}-\frac{1}{4} w_{x x}^{n}\right)+1\right] w^{n+1}} \\
& \quad-\Delta t\left[w_{x}^{n}-\left(w^{n}\right)^{2}\right] w_{x}^{n+1}-\frac{\Delta t}{4} w^{n} w_{x x}^{n+1} \\
& \quad=w^{n}\left[1+\Delta t w^{n} w_{x}^{n}\right]
\end{aligned}
$$

for $n=0,1, \ldots$. To start computations in Eq. (8), we need $w^{0}=w_{0}(x)$, which is obtained from the initial condition in Eq. (3). Moreover, the boundary conditions obtained from Eq. (4) at $x=0, L$ are:

$$
\begin{aligned}
& w^{n+1}(0):=h_{0}^{n+1}=h_{0}\left(t_{n+1}\right), \\
& w^{n+1}(L):=h_{1}^{n+1}=h_{1}\left(t_{n+1}\right), \quad n=0,1, \ldots, M-1 .
\end{aligned}
$$

\section{Bessel functions: Basic matrix relations}

Now, the first stage in discretizing the foam drainage 
equation in time is carried out through Eq. (8). In the second stage, it is required to approximate the solution of the original model (1) with respect to the space variable through solving Eq. (8). To do so, it is assumed that the solution $w^{n+1}$ of Eq. (8) can be written as a combination of $\mathbb{B}_{\ell}(x)$. In the first time step, i.e., for $n=0$, we use the initial condition $w_{0}(x)$ to determine $w^{0}$ exactly. Let $\mathcal{W}_{n, N}(x)$ denotes the approximate solution of $w^{n}$ at time level $t_{n}$. Then, the $\mathcal{W}_{n+1, N}(x)$ is sought after at the next time level $t_{n+1}$ as follows:

$$
\mathcal{W}_{n+1, N}(x)=\sum_{\ell=0}^{N} a_{\ell, n} \mathbb{B}_{\ell}(x), \quad x \in[0, L],
$$

for $n=0,1, \ldots, M-1$. Here, $a_{\ell, n}, \ell=0,1, \ldots, N$ as the unknown Bessel coefficients must be found. Let us introduce the Bessel vector $\mathbf{B}_{N}(x)$ as well as the unknown vector $\mathbf{A}_{n, N}$ in the forms:

$$
\begin{aligned}
& \mathbf{B}_{N}(x)=\left[\begin{array}{llll}
\mathbb{B}_{0}(x) & \mathbb{B}_{1}(x) & \ldots & \mathbb{B}_{N}(x)
\end{array}\right], \\
& \mathbf{A}_{n, N}=\left[\begin{array}{llll}
a_{0, n} & a_{1, n} & \ldots & a_{n, N}
\end{array}\right]^{T} .
\end{aligned}
$$

With the help of these vectors, we are able to rewrite Eq. (10) in a compact representation as follows:

$$
\mathcal{W}_{n+1, N}(x)=\mathbf{B}_{N}(x) \mathbf{A}_{n, N},
$$

additionally, by introducing the matrix $\mathbf{D}$ shown in Box I, and the monomial vector $\mathbf{X}_{N}(x)=$ $\left[\begin{array}{lllll}1 & x & x^{2} & \ldots & x^{N}\end{array}\right]$, we shall express $\mathbf{B}_{N}(x)$ as follows:

$$
\mathbf{B}_{N}(x)=\mathbf{X}_{N}(x) \mathbf{D}^{T} \text {. }
$$

We are left with finding a relationship between $\mathbf{X}_{N}(x)$ and $\frac{d}{d x} \mathbf{X}_{N}(x)$. A straightforward calculation shows that:

$$
\frac{d}{d x} \mathbf{X}_{N}(x)=\mathbf{X}_{N}(x) \mathbf{M}^{T}
$$

$$
\mathbf{M}^{T}=\left[\begin{array}{ccccc}
0 & 1 & 0 & \ldots & 0 \\
0 & 0 & 2 & \ldots & 0 \\
\vdots & \vdots & \ddots & \vdots & \vdots \\
0 & 0 & 0 & \ddots & N \\
0 & 0 & 0 & \ldots & 0
\end{array}\right]_{(N+1) \times(N+1)}
$$

We consider the result of the convergence of the Bessel functions as $N \rightarrow \infty$. This property indicates that the Bessel function is exponentially convergent in the weighted $L_{2}$ norm with respect to weight function $r(x)=\exp (-2 L / x)$.

Theorem $3.1[22]$ Let $Z_{N}(x)=\mathbf{B}_{N}(x) \mathbf{A}_{n, N}$ be the best square approximation to $Z(x)$. Under the assumptions $Z(x) \in \mathcal{C}^{N+1}[0, L]$ and $M_{\infty}:=$ $\max _{x \in[0, L]}\left|Z^{(N+1)}(x)\right|$, we have the following error bound:

$$
\left\|Z(x)-Z_{N}(x)\right\|_{r} \leq \frac{M_{\infty}}{\sqrt{2 N+3}} \frac{L^{M+\frac{3}{2}}}{(N+1) !} \frac{1}{\exp (1)} .
$$

\section{Taylor-Bessel collocation method}

Now, the solution to the discretized model problem (8) is to be approximated via Eq. (10). To do so, we first define the set of collocation points $\left\{x_{q}\right\}_{q=0}^{N}$ on $[0, L]$ with:

$$
x_{q}=\frac{L}{N} q, \quad q=0,1, \ldots, N .
$$

Next, we express the unknown functions $w^{n+1}, w_{x}^{n+1}$, and $w_{x x}^{n+1}$ in Eq. (8) in a matrix form. By placing the collocation points (Eq. (14)) into the resulting equation, we get a linear matrix equation.

Our next task is to combine two previously asserted Relations (Eqs. (11) and (12)). In this way, Eq. (10) is rewritten in the matrix expression as follows:

$$
\mathcal{W}_{n+1, N}(x)=\mathbf{X}_{N}(x) \mathbf{D}^{T} \mathbf{A}_{n, N} .
$$

$$
\mathbf{D}=\left[\begin{array}{cccccc}
1 & 0 & 0 & \cdots & 0 & 0 \\
1 & 1 & 0 & \cdots & 0 & 0 \\
1 & 3 & 3 & \cdots & 0 & 0 \\
\vdots & \vdots & \ddots & \ddots & \ddots & \vdots \\
1 & \frac{N !}{(N-2) ! 1 ! 2^{1}} & \frac{(N+1) !}{(N-3) ! 2 ! 2^{2}} & \cdots & \frac{(2 N-2) !}{0 !(N-1) ! 2^{N-1}} & 0 \\
1 & \frac{(N+1) !}{(N-1) ! 1 ! 2^{1}} & \frac{(N+2) !}{(N-2) ! 2 ! 2^{2}} & \cdots & \frac{(2 N-1) !}{1 !(N-1) ! 2^{N-1}} & \frac{(2 N) !}{0 ! N ! 2^{N}}
\end{array}\right]_{(N+1) \times(N+1)}
$$


After evaluating the preceding equation at the collocation points (Eq. (14)) we arrive at:

$$
\begin{aligned}
& \mathbf{W}_{n+1}=\mathbf{Y ~ D}^{T} \mathbf{A}_{n, N}, \quad \mathbf{W}_{n+1}=\left[\begin{array}{c}
\mathcal{W}_{n+1, N}\left(x_{0}\right) \\
\mathcal{W}_{n+1, N}\left(x_{1}\right) \\
\vdots \\
\mathcal{W}_{n+1, N}\left(x_{N}\right)
\end{array}\right], \\
& \mathbf{Y}=\left[\begin{array}{c}
\mathbf{X}_{N}\left(x_{0}\right) \\
\mathbf{X}_{N}\left(x_{1}\right) \\
\vdots \\
\mathbf{X}_{N}\left(x_{N}\right)
\end{array}\right] .
\end{aligned}
$$

We can represent the first and second-orders derivatives in Eq. (8) through Eqs. (13) and (15) in the matrix forms:

$$
\left\{\begin{array}{l}
w_{x}^{n+1} \approx \mathcal{W}_{n+1, N}^{(1)}(x)=\mathbf{X}_{N}(x) \mathbf{M}^{T} \mathbf{D}^{T} \mathbf{A}_{n, N}, \\
w_{x x}^{n+1} \approx \mathcal{W}_{n+1, N}^{(2)}(x)=\mathbf{X}_{N}(x)\left(\mathbf{M}^{T}\right)^{2} \mathbf{D}^{T} \mathbf{A}_{n, N} .
\end{array}\right.
$$

Similarly, by evaluating them at the collocation points, the first and second derivatives in Eqs. (17) can be written in the matrix forms:

$$
\begin{aligned}
\dot{\mathbf{W}}_{n+1} & =\mathbf{Y} \mathbf{M}^{T} \mathbf{D}^{T} \mathbf{A}_{n, N}, \\
\dot{\mathbf{W}}_{n+1} & =\left[\begin{array}{c}
\mathcal{W}_{n+1, N}^{(1)}\left(x_{0}\right) \\
\mathcal{W}_{n+1, N}^{(1)}\left(x_{1}\right) \\
\vdots \\
\mathcal{W}_{n+1, N}^{(1)}\left(x_{N}\right)
\end{array}\right], \\
\ddot{\mathbf{W}}_{n+1}= & \mathbf{Y}\left(\mathbf{M}^{T}\right)^{2} \mathbf{D}^{T} \mathbf{A}_{n, N}, \\
\ddot{\mathbf{W}}_{n+1}= & {\left[\begin{array}{c}
\mathcal{W}_{n+1, N}^{(2)}\left(x_{0}\right) \\
\mathcal{W}_{n+1, N}^{(2)}\left(x_{1}\right) \\
\vdots \\
\mathcal{W}_{n+1, N}^{(2)}\left(x_{N}\right)
\end{array}\right] . }
\end{aligned}
$$

By introducing the following functions:

$$
\begin{aligned}
& p_{n, 0}(x)=2 \Delta t w^{n} w_{x}^{n}-\frac{1}{4} \Delta t w_{x x}^{n}+1, \\
& p_{n, 1}(x)=-\Delta t w_{x}^{n}+\Delta t\left(w^{n}\right)^{2}, \\
& p_{n, 2}(x)=-\frac{1}{4} \Delta t w^{n} \\
& g_{n}(x)=w^{n}+\Delta t\left(w^{n}\right)^{2} w_{x}^{n},
\end{aligned}
$$

and using the approximations $\mathcal{W}_{n+1, N}(x), \mathcal{W}_{n+1, N}^{(1)}(x)$, $\mathcal{W}_{n+1, N}^{(2)}(x)$, we may rewrite Eq. (8) as:

$$
\begin{aligned}
p_{n, 2}(x) & \mathcal{W}_{n+1, N}^{(2)}(x)+p_{n, 1}(x) \mathcal{W}_{n+1, N}^{(1)}(x) \\
& +p_{n, 0}(x) \mathcal{W}_{n+1, N}(x)=g_{n}(x), \quad 0 \leq x \leq L .
\end{aligned}
$$

By inserting the collocation points into Eq. (20), the following system is obtained:

$$
\mathbf{P}_{n, 2} \ddot{\mathbf{W}}_{n+1}+\mathbf{P}_{n, 1} \dot{\mathbf{W}}_{n+1}+\mathbf{P}_{n, 0} \mathbf{W}_{n+1}=\mathbf{G}_{n} .
$$

In Eq. (21), the matrices $\mathbf{P}_{n, l}$, and the vector $\mathbf{G}_{n}$ take the forms:

$$
\mathbf{P}_{n, l}=\left[\begin{array}{cccc}
p_{n, l}\left(x_{0}\right) & 0 & \ldots & 0 \\
0 & p_{n, l}\left(x_{1}\right) & \cdots & 0 \\
\vdots & \vdots & \ddots & \vdots \\
0 & 0 & \cdots & p_{n, l}\left(x_{N}\right)
\end{array}\right]_{(N+1) \times(N+1)}
$$

$$
\mathbf{G}_{n}=\left[\begin{array}{c}
g_{n}\left(x_{0}\right) \\
g_{n}\left(x_{1}\right) \\
\vdots \\
g_{n}\left(x_{N}\right)
\end{array}\right]_{(N+1) \times 1}
$$

$\ell=0,1,2$. Let's place Eqs. (16), (18), and (19) into Eq. (21). This yields the fundamental matrix equation:

$$
\mathbf{V}_{n} \mathbf{A}_{n, N}=\mathbf{G}_{n},
$$

where:

$$
\mathbf{V}_{n}:=\left\{\mathbf{P}_{n, 2} \mathbf{Y}\left(\mathbf{M}^{T}\right)^{2}+\mathbf{P}_{n, 1} \mathbf{Y} \mathbf{M}^{T}+\mathbf{P}_{n, 0} \mathbf{Y}\right\} \mathbf{D}^{T} .
$$

Clearly, the fundamental matrix equation (Eq. (22)) is a set of $(N+1)$ linear equations in terms of $(N+1)$ unknown coefficients $a_{0, n}, a_{1, n}, \ldots, a_{N, n}$ to be found.

To consider the boundary conditions (Eq. (9)), we must also convert them into matrix form. Based on the representation (Eq. (15)), these conditions, i.e. $\mathcal{W}_{n+1 . N}(0)=h_{0}^{n+1}$ and $\mathcal{W}_{n+1, N}(1)=h_{1}^{n+1}$, can be expressed in the matrix notation:

$$
\begin{aligned}
& \widehat{\mathbf{V}}_{n, 0} \mathbf{A}_{n, N}=h_{0}^{n+1}, \\
& \widehat{\mathbf{V}}_{n, 0}:=\mathbf{X}_{N}(0) \mathbf{D}^{T}=\left[\begin{array}{llll}
\hat{v}_{0,0} & \hat{v}_{0,1} & \ldots & \hat{v}_{0, N}
\end{array}\right], \\
& \widehat{\mathbf{V}}_{n, 1} \mathbf{A}_{n, N}=h_{1}^{n+1}, \\
& \widehat{\mathbf{V}}_{n, 1}:=\mathbf{X}_{N}(1) \mathbf{D}^{T}=\left[\begin{array}{llll}
\hat{v}_{1,0} & \hat{v}_{1,1} & \ldots & \hat{v}_{1, N}
\end{array}\right] .
\end{aligned}
$$

Next, we substitute the first two rows of the augmented matrix $\left[\mathbf{V}_{n} ; \mathbf{G}_{n}\right]$ by the vectors $\left[\widehat{\mathbf{V}}_{n, 0} ; h_{0}^{n+1}\right]$ and $\left[\widehat{\mathbf{V}}_{n, 1} ; h_{1}^{n+1}\right]$, for convenience. Thus, the modified linear system of equations is obtained by Eq. (23) as shown in Box II. Now, by solving the above linear system, we may to obtain the unknown Bessel coefficients in Eq. (15).

\section{Numerical simulations}

To testify the performance of the combined Taylor and Bessel-collocation approach, numerical simulations 


$$
\left[\widehat{\mathbf{V}}_{n} ; \widehat{\mathbf{G}}_{n}\right]=\left[\begin{array}{llllllll}
\hat{v}_{0,0} & \hat{v}_{0,1} & \hat{v}_{0,2} & \hat{v}_{0,3} & \ldots & \hat{v}_{0, N} & ; & h_{0}^{n+1} \\
\hat{v}_{1,0} & \hat{v}_{1,1} & \hat{v}_{1,2} & \hat{v}_{1,3} & \ldots & \hat{v}_{1, N} & ; & h_{1}^{n+1} \\
v_{2,0} & v_{2,1} & v_{2,2} & v_{2,3} & \ldots & v_{2, N} & ; & g_{n}\left(x_{2}\right) \\
\vdots & \vdots & \vdots & \ddots & \vdots & \vdots & \vdots \\
v_{N-1,0} & v_{N-1,1} & v_{N-1,2} & v_{N-1,3} & \ldots & v_{N-1, N} & ; & g_{n}\left(x_{N-1}\right) \\
v_{N, 0} & v_{N, 1} & v_{N, 2} & v_{N, 3} & \ldots & v_{N, N} & ; & g_{n}\left(x_{N}\right)
\end{array}\right]
$$

based on two test cases are given for the nonlinear initial and boundary value Problems (Eqs. (3) and (4)). Furthermore, comparisons of numerical results and the outcomes of diverse existing schemes are also made for validation. For implementations, MATLAB software (version 2017a) is employed.

Test problem 5.1. We first consider the foam drainage equation (Eq. (3)) with the following initial condition $[4,24,11]$ :

$$
w_{0}(x)=-\tanh (x) .
$$

The exact solution is given by $w(x, t)=-\tanh (x-t)$.

We first employ $\Delta t$ equal to $T=0.01$. Considering Eq. (10) with $N=5$, the following approximation for $0 \leq x \leq L=1$ is obtained:

$$
\begin{aligned}
\mathcal{W}_{1,5}(x)= & 0.01807890048 x^{5}-0.1934987886 x^{4} \\
& +0.4424756341 x^{3}-0.03689672509 x^{2} \\
& -0.9975210119 x+0.00999966668 .
\end{aligned}
$$

We plot the above obtained solution as an approximation to $w(x, T)$ in Figure 1. We also show the corresponding Absolute Errors (AE) $\left|w(x, T)-\mathcal{W}_{1,5}(x)\right|$

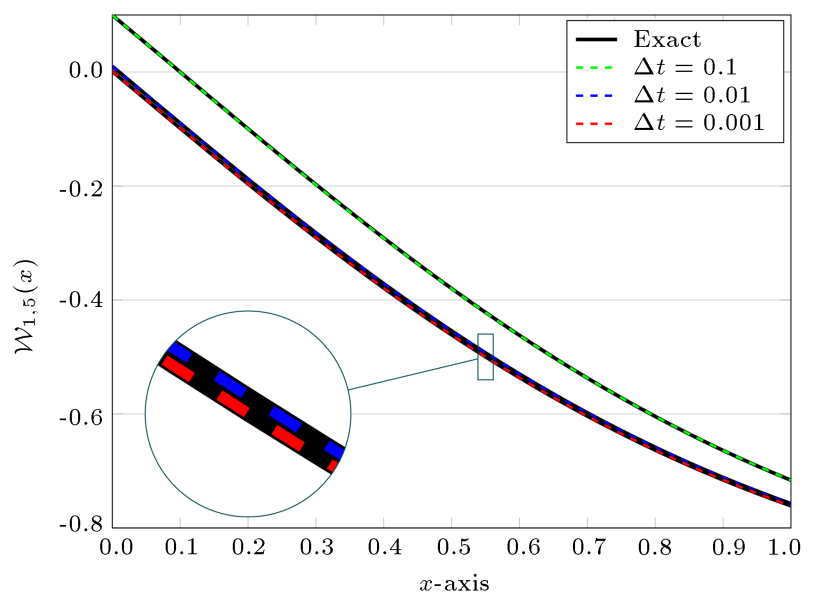

Figure 1. Graphs of exact and solutions at different time instants $t=\Delta t$ for $\Delta t=0.1,0.01,0.001, N=5$ in test problem 5.1.

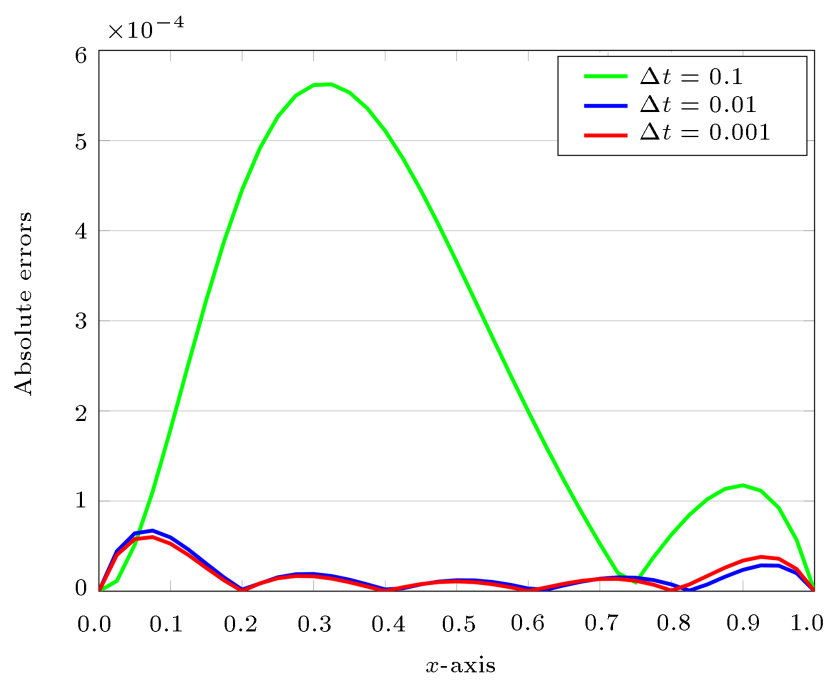

Figure 2. Graphs of absolute errors at different time instants $t=\Delta t$ for $\Delta t=0.1,0.01,0.001, N=5$ in test problem 5.1.

at $x \in[0,1]$ in Figure 2. Besides $\Delta t=0.01$, we use $\Delta t=0.1,0.01$ to demonstrate the impact of different values of time step size on the computations.

To validate our results, some comparisons are made in Tables 1 and 2, which show the numerical solutions obtained by the presented scheme evaluated at $t=0.01,0.001$ and various $x \in[0,1]$. The corresponding $\mathrm{AE}$ are also reported in the second column of these tables. Furthermore, analogue results of the previously well-established methods are displayed in Table 1. These include the collocation method based on Bivariate Chebyshev Functions (BCF) [11], the Adomian Decomposition Method (ADM) [4], the Homotopy Perturbation Method (HPM) [5], the Haar Wavelet Quasilinearization Approach (HWQA) [24], and the Homotopy Perturbation Transform Method (HPTM) [10]. It can be observed that our numerical results are in good agreement with the corresponding exact solutions. However, our approach is more straightforward than other existing methods.

Test problem 5.2. As the second example, we consider the following initial condition [4,24,11]: 
Table 1. The comparison of numerical results in Test Problem 5.1 for $N=8$ and various $x \in[0,1]$ at time $t=0.01$.

\begin{tabular}{cccccccc}
\hline$\frac{\boldsymbol{x}}{64}$ & Present & AE & BCF [11] & HWQA [24] & ADM [4] & HPM [5] & HPTM [10] \\
\hline 1 & -0.0056255222 & $5.82_{-7}$ & -0.005624 & -0.005626 & -0.004253 & -0.004358 & -0.048341 \\
3 & -0.0368592824 & $9.87_{-7}$ & -0.036858 & -0.036874 & -0.009002 & -0.027011 & -0.017108 \\
5 & -0.0680207213 & $9.16_{-7}$ & -0.068019 & -0.068085 & -0.015399 & -0.058439 & -0.014053 \\
7 & -0.0990498931 & $7.28_{-7}$ & -0.099049 & -0.099199 & -0.023360 & -0.089857 & -0.045083 \\
9 & -0.1298876683 & $5.79_{-7}$ & -0.129887 & -0.130158 & -0.032796 & -0.121194 & -0.075921 \\
27 & -0.3900638141 & $2.53_{-7}$ & -0.390063 & -0.392716 & -0.168432 & -0.387936 & -0.336218 \\
29 & -0.4162316016 & $2.05_{-7}$ & -0.416231 & -0.419230 & -0.187449 & -0.414778 & -0.362463 \\
31 & -0.4417276182 & $1.71_{-7}$ & -0.441727 & -0.445063 & -0.206944 & -0.440899 & -0.388074 \\
33 & -0.4665295588 & $1.49_{-7}$ & -0.466529 & -0.470208 & -0.226840 & -0.466274 & -0.413043 \\
35 & -0.4906189456 & $1.30_{-7}$ & -0.490618 & -0.494646 & -0.247065 & -0.490881 & -0.437369 \\
55 & -0.6907427887 & $2.57_{-8}$ & -0.690742 & -0.697478 & -0.453650 & -0.693486 & -0.651564 \\
57 & -0.7067322567 & $3.28_{-8}$ & -0.706732 & -0.713630 & -0.473606 & -0.709521 & -0.671453 \\
59 & -0.7220308937 & $8.46_{-8}$ & -0.722030 & -0.729056 & -0.493271 & -0.724843 & -0.691471 \\
61 & -0.7366545782 & $1.55_{-7}$ & -0.736654 & -0.743775 & -0.512612 & -0.739469 & -0.711761 \\
63 & -0.7506204287 & $1.27-7$ & -0.750620 & -0.757808 & -0.531603 & -0.753420 & -0.732485 \\
\hline
\end{tabular}

Table 2. The comparison of numerical results in Test problem 5.1 for $N=8$ and various $x \in[0,1]$ at time $t=0.001$.

\begin{tabular}{cccccccc}
\hline$\frac{\boldsymbol{x}}{\mathbf{6 4}}$ & Present & AE & BCF [11] & HWQA [24] & ADM [4] & HPM [5] & HPTM [10] \\
\hline 1 & -0.0146242559 & $2.99_{-7}$ & -0.014624 & -0.014624 & -0.000433 & -0.013626 & -0.039344 \\
3 & -0.0458432514 & $4.06_{-7}$ & -0.045843 & -0.045847 & -0.002700 & -0.044858 & -0.008125 \\
5 & -0.0769726894 & $2.46_{-7}$ & -0.076972 & -0.076984 & -0.006885 & -0.076014 & -0.023004 \\
7 & -0.1079527574 & $6.67_{-8}$ & -0.107953 & -0.107973 & -0.012948 & -0.107033 & -0.053984 \\
9 & -0.1387246409 & $4.28_{-8}$ & -0.138725 & -0.138769 & -0.020837 & -0.137855 & -0.084756 \\
27 & -0.3976673000 & $1.64_{-8}$ & -0.397667 & -0.398034 & -0.160042 & -0.397455 & -0.343819 \\
29 & -0.4236441749 & $1.58_{-8}$ & -0.423644 & -0.423939 & -0.181144 & -0.423499 & -0.369872 \\
31 & -0.4489424537 & $6.57_{-9}$ & -0.448942 & -0.449301 & -0.202947 & -0.448860 & -0.395283 \\
33 & -0.4735409398 & $6.06_{-9}$ & -0.473541 & -0.473964 & -0.225333 & -0.473515 & -0.420046 \\
35 & -0.4974222452 & $1.56_{-8}$ & -0.497422 & -0.497826 & -0.248187 & -0.497448 & -0.444159 \\
55 & -0.6954195363 & $5.22_{-8}$ & -0.695419 & -0.696107 & -0.480881 & -0.695694 & -0.655872 \\
57 & -0.7112084249 & $3.79_{-8}$ & -0.711208 & -0.711883 & -0.502829 & -0.711487 & -0.675449 \\
59 & -0.7263108975 & $1.93_{-7}$ & -0.726311 & -0.726999 & -0.524303 & -0.726592 & -0.695130 \\
61 & -0.7407432348 & $3.37-7$ & -0.740744 & -0.741464 & -0.545268 & -0.741025 & -0.715055 \\
63 & -0.7545229508 & $2.54_{-7}$ & -0.754523 & -0.755247 & -0.565692 & -0.754803 & -0.735378 \\
\hline
\end{tabular}

$$
w_{0}(x)=\left(1+e^{x}\right)^{-1}-\frac{1}{2} .
$$

It is shown that the exact solution is given by $w(x, t)=$ $\left(1+e^{x-\frac{t}{4}}\right)^{-1}-\frac{1}{2}$.

For this test problem, we consider $T=0.1$, $\Delta t=0.001$, and $N=5$. The snapshots of numerical solutions at different time instants $t=s \Delta t, s=$ $1,2, \ldots, 100$ are shown in Figure 3. In addition, the corresponding $\mathrm{AE}$ are also plotted in Figure 4. In this case, the approximate solutions at $t=\Delta t$ and $t=T$ are obtained as follows:

$$
\begin{aligned}
\mathcal{W}_{1,5}(x) & =-0.001109924646 x^{5}-0.001404371504 x^{4} \\
& +0.02164565497 x^{3}-0.0002217269879 x^{2} \\
& -0.2499815546 x+0.00006249999967
\end{aligned}
$$


Table 3. The comparison of $L_{2}$ and $L_{\infty}$ error norms in Test problem 5.2 for diverse $N=4,5, \ldots, 8, \Delta t=0.001,0.01,0.1$ evaluated at the final times $t=T$ with $T=0.1,0.5,1$.

\begin{tabular}{ccccccccc}
\hline & \multicolumn{4}{c}{$\boldsymbol{\Delta} \boldsymbol{t}=\mathbf{0 . 0 0 1}$} & \multicolumn{2}{c}{$\boldsymbol{\Delta} \boldsymbol{t}=\mathbf{0 . 0 1}$} & \multicolumn{2}{c}{$\boldsymbol{\Delta t}=\mathbf{0 . 1}$} \\
\cline { 2 - 9 } $\boldsymbol{N}$ & \multicolumn{2}{c}{$\boldsymbol{T}=\mathbf{0 . 1}$} & \multicolumn{2}{c}{$\boldsymbol{T}=\mathbf{0 . 5}$} & \multicolumn{2}{c}{$\boldsymbol{T}=\mathbf{1}$} & \multicolumn{2}{c}{$\boldsymbol{T}=\mathbf{1}$} \\
\cline { 2 - 9 } & $\boldsymbol{L}_{\infty}$ & $\boldsymbol{L}_{\mathbf{2}}$ & $\boldsymbol{L}_{\boldsymbol{\infty}}$ & $\boldsymbol{L}_{\mathbf{2}}$ & $\boldsymbol{L}_{\boldsymbol{\infty}}$ & $\boldsymbol{L}_{\mathbf{2}}$ & $\boldsymbol{L}_{\boldsymbol{\infty}}$ & $\boldsymbol{L}_{\mathbf{2}}$ \\
\hline 4 & $6.137_{-6}$ & $1.533_{-6}$ & $1.534_{-4}$ & $2.034_{-5}$ & $6.512_{-4}$ & $3.349_{-4}$ & $5.859_{-4}$ & $2.966_{-4}$ \\
5 & $6.264_{-6}$ & $2.606_{-7}$ & $1.607_{-4}$ & $4.955_{-5}$ & $1.133_{-3}$ & $5.212_{-4}$ & $9.678_{-4}$ & $4.496_{-4}$ \\
6 & $5.918_{-6}$ & $2.316_{-7}$ & $1.391_{-4}$ & $5.211_{-5}$ & $1.577_{-3}$ & $7.531_{-4}$ & $9.771_{-4}$ & $5.598_{-4}$ \\
7 & $6.033_{-6}$ & $6.316_{-8}$ & $2.164_{-4}$ & $7.232_{-5}$ & $3.833_{-3}$ & $1.668_{-3}$ & $2.514_{-3}$ & $9.270_{-4}$ \\
8 & $6.076_{-6}$ & $2.276_{-7}$ & $3.382_{-4}$ & $9.884_{-5}$ & $1.249_{-3}$ & $4.766_{-3}$ & $1.919_{-3}$ & $1.041_{-3}$ \\
\hline
\end{tabular}

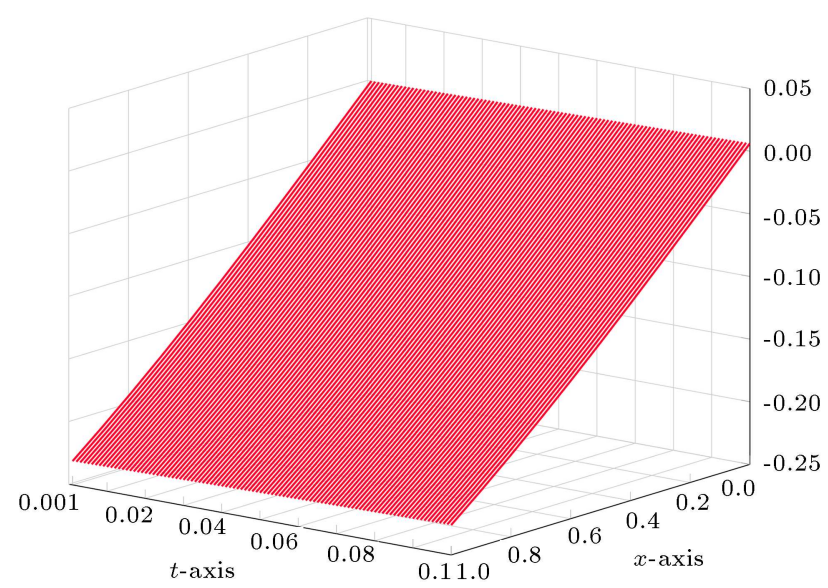

Figure 3. Graphs of numerical solutions in Test problem 5.2 at different time instants $t=s \Delta t, s=1,2$, $\ldots, 100$ for $\Delta t=0.001, T=0.1$, and $N=5$.

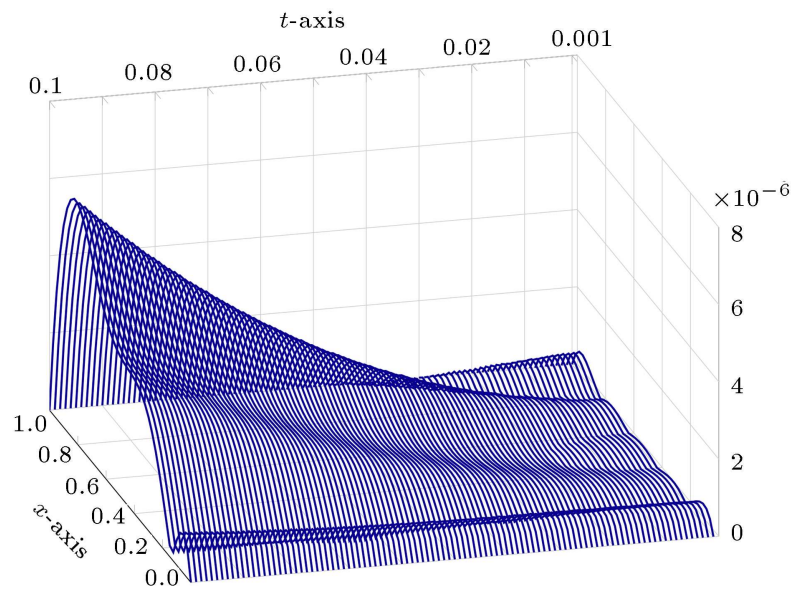

Figure 4. Graphs of absolute errors in Test problem 5.2 at different time instants $t=s \Delta t, s=1,2, \ldots, 100$ for $\Delta t=0.001, T=0.1$, and $N=5$.

$$
\begin{aligned}
\mathcal{W}_{100,5}(x) & =-0.000731548639 x^{5}-0.002111324555 x^{4} \\
& +0.0224345299 x^{3}-0.002035416473 x^{2} \\
& -0.249920896 x+0.0062496745
\end{aligned}
$$

We next compute the maximum $\mathrm{AE}$ which are denoted by $L_{\infty}$ and $L_{2}$ error norms evaluated at the final time $t=T$ via:

$$
\begin{aligned}
L_{\infty}: & : \max _{0 \leq x \leq 1}\left|w(x, T)-\mathcal{W}_{M+1, N}(x)\right|, \\
L_{2}:= & \left(\frac{1}{N+1} \int_{0}^{1}[w(x, T)\right. \\
& \left.\left.-\mathcal{W}_{M+1, N}(x)\right]^{2} d x\right)^{\frac{1}{2}} .
\end{aligned}
$$

We utilize various $N=4,5, \ldots, 8$ and report the results of errors in Table 3. Also, different final times $T=$ $0.1,0.5$, and $T=1$ are used with the step sizes $\Delta t=$ $0.001,0.001$, and $T=0.1$.

Finally, our numerical results and computations are verified through a comparison with well-established numerical models and simulations. Tables 4 and 5 show these comparisons with the methods used in Tables 1 and 2 in Test problem 5.1. However, in Tables 4 and 5, the Laplace Decomposition Method (LDM) [25] is employed rather than HPM.

\section{Conclusions}

In this work, a space and time-accurate approximation technique was presented to solve the foam drainage equation. For the temporal discretization, the Taylor series expansion approach with order $O\left(\Delta t^{2}\right)$ was employed. Afterwards, at each time step, the novel Bessel based collocation approach with exponential accuracy was utilized to approximate the space variable. By using the matrix representations of these polynomials in conjunction with the collocation points, the scheme converts the underlying model problem into an algebraic linear system of equations. The utility and accuracy of the presented technique were examined by using numerical experiments. Comparisons with earlier computational and experimental studies were also made. The presented results demonstrated the reliability and the applicability of the presented combined algorithm for the nonlinear time-dependent foam drainage equation. The combined technique with 
Table 4. The comparison of numerical results in Test problem 5.2 for $N=8$ and various $x \in[0,1]$ at time $t=0.1$.

\begin{tabular}{cccccccc}
\hline$\frac{\boldsymbol{x}}{64}$ & Present & AE & BCF [11] & HWQA [24] & ADM [4] & HPM [5] & HPTM [10] \\
\hline 1 & +0.0023435127 & $2.20_{-7}$ & +0.002344 & +0.002344 & +0.002098 & +0.002083 & +0.002084 \\
3 & -0.0054690211 & $4.89_{-7}$ & -0.005469 & -0.005468 & -0.005793 & -0.005785 & -0.005787 \\
5 & -0.0132787383 & $6.11_{-7}$ & -0.013278 & -0.013278 & -0.013664 & -0.013650 & -0.013651 \\
7 & -0.0210818974 & $6.53_{-7}$ & -0.021081 & -0.021081 & -0.021512 & -0.021508 & -0.021509 \\
9 & -0.0288747443 & $6.56_{-7}$ & -0.028874 & -0.028874 & -0.029357 & -0.029354 & -0.029353 \\
27 & -0.0979371596 & $5.45_{-7}$ & -0.097937 & -0.097935 & -0.098832 & -0.098830 & -0.098831 \\
29 & -0.1054263741 & $5.27_{-7}$ & -0.105426 & -0.105424 & -0.106358 & -0.106357 & -0.106358 \\
31 & -0.1128664061 & $5.08_{-7}$ & -0.112866 & -0.112863 & -0.113834 & -0.113833 & -0.113833 \\
33 & -0.1202541435 & $4.88_{-7}$ & -0.120254 & -0.120251 & -0.121255 & -0.121254 & -0.121254 \\
35 & -0.1275865681 & $4.69_{-7}$ & -0.127586 & -0.127583 & -0.128620 & -0.128619 & -0.128619 \\
55 & -0.1972794716 & $2.66_{-7}$ & -0.197279 & -0.197274 & -0.198532 & -0.198532 & -0.198532 \\
57 & -0.2038347870 & $2.50_{-7}$ & -0.203834 & -0.203830 & -0.205099 & -0.205099 & -0.205099 \\
59 & -0.2103071180 & $2.27_{-7}$ & -0.210307 & -0.210304 & -0.211582 & -0.211582 & -0.211582 \\
61 & -0.2166949149 & $1.81_{-7}$ & -0.216695 & -0.216691 & -0.217979 & -0.217979 & -0.217979 \\
63 & -0.2229967352 & $8.29_{-8}$ & -0.222997 & -0.222994 & -0.224288 & -0.224288 & -0.224288 \\
\hline
\end{tabular}

Table 5. The comparison of numerical results in Test problem 5.2 for $N=8$ and various $x \in[0,1]$ at time $t=0.01$.

\begin{tabular}{cccccccc}
\hline$\frac{\boldsymbol{x}}{\mathbf{6 4}}$ & Present & AE & BCF [11] & HWQA [24] & ADM [4] & HPM [5] & HPTM [10] \\
\hline 1 & -0.0032812030 & $1.18_{-10}$ & -0.003281 & -0.003281 & -0.003309 & -0.003307 & -0.003307 \\
3 & -0.0110919303 & $3.50_{-10}$ & -0.011092 & -0.011091 & -0.011126 & -0.011123 & -0.011123 \\
5 & -0.0188972450 & $5.21_{-10}$ & -0.018897 & -0.018897 & -0.018939 & -0.018935 & -0.018934 \\
7 & -0.0266933472 & $6.16_{-10}$ & -0.026693 & -0.026693 & -0.026741 & -0.026737 & -0.026736 \\
9 & -0.0344764548 & $6.52_{-10}$ & -0.034476 & -0.034476 & -0.034529 & -0.034525 & -0.034524 \\
27 & -0.1033336837 & $5.28_{-10}$ & -0.103334 & -0.103333 & -0.103430 & -0.103424 & -0.103423 \\
29 & -0.1107878235 & $5.10_{-10}$ & -0.110788 & -0.110787 & -0.110888 & -0.110883 & -0.110880 \\
31 & -0.1181905313 & $4.91_{-10}$ & -0.118191 & -0.118190 & -0.118297 & -0.118289 & -0.118287 \\
33 & -0.1255387617 & $4.71_{-10}$ & -0.125539 & -0.125538 & -0.125649 & -0.125641 & -0.125638 \\
35 & -0.1328295665 & $4.52_{-10}$ & -0.132830 & -0.132829 & -0.132943 & -0.132932 & -0.132932 \\
55 & -0.2020073393 & $2.59_{-10}$ & -0.202007 & -0.202007 & -0.202149 & -0.202136 & -0.202132 \\
57 & -0.2085030871 & $2.27_{-10}$ & -0.208503 & -0.208502 & -0.208650 & -0.208636 & -0.208629 \\
59 & -0.2149147407 & $1.71_{-10}$ & -0.214915 & -0.214914 & -0.215065 & -0.215047 & -0.215041 \\
61 & -0.2212408533 & $9.18_{-11}$ & -0.221241 & -0.221240 & -0.221389 & -0.221376 & -0.221368 \\
63 & -0.2274800989 & $1.51_{-11}$ & -0.227480 & -0.227480 & -0.227639 & -0.227618 & -0.227608 \\
\hline
\end{tabular}

inherited simplicity and ease of implementation can be easily extended to other nonlinear model problems in diverse disciplines of engineering and sciences.

\section{Nomenclature}

$\begin{array}{ll}\text { AE } & \text { Absolute Errors } \\ \text { BCF } & \text { Bivariate Chebyshev Functions } \\ \text { ADM } & \text { Adomian Decomposition Method } \\ \text { HPM } & \text { Homotopy Perturbation Method }\end{array}$

HWQA Haar Wavelet Quasilinearization Approach

HPTM Homotopy Perturbation Transform Method

LDM Laplace Decomposition Method

\section{References}

1. Verbist, G., Weaire, D., and Kraynik, A.M. "The foam drainage equation", J. Phys. Condens. Matter, 83, pp. 715-731 (1996).

2. Prud'homme, R.K. and Khan S.A. (Eds.), Foams: 
Theory, Measurements and Applications, Dekker, New York (1996).

3. Weaire, D.L. and Hutzler, S., The Physics of Foams, Oxford University Press, Oxford (2000).

4. Helal, M.A. and Mehanna, M.S. "The tanh method and Adomian decomposition method for solving the foam drainage equation", Appl. Math. Comput., 190, pp. 599-609 (2007).

5. Fereidoon, A.H., Yaghoobi, H., and Davoudabadi, M.R. "Application of the homotopy perturbation method for solving the foam drainage equation", Int. J. Differ. Equ., 2011, Article ID 864023 (2011).

6. Yașar, E. and Özer, T. "On symmetries, conservation laws, and invariant solutions of the foam-drainage equation", Int. J. Nonlin. Mech., 46(2), pp. 357-362 (2011).

7. Nadjafikhah, M. and Chekini, O. "Conservation law and lie symmetry analysis of foam-drainage equation", AUT J. Math. Com., 2(1), pp. 37-44 (2021).

8. Darvishi, M.T. and Khani, F. "A series solution of the foam drainage equation", Comput. Math. Appl., 58, pp. 360-368 (2009).

9. Khani, F., Hamedi-Nezhad, S., Darvishi M.T., et al. "New solitary wave and periodic solutions of the foam drainage equation using the exp-function method", Nonlinear Anal.: Real World Appl., 10, pp. 1904-1911 (2009).

10. Khan, Y. "A method for solving nonlinear timedependent drainage model", Neural Comput. and Applic., 23, pp. 411-415 (2013).

11. Parand, K. and Delkhosh, M. "An efficient numerical method for solving nonlinear foam drainage equation", Indian J. Phys., 92(2), pp. 231-243 (2018).

12. Wang, L. and Qian, Z. "A meshfree stabilized collocation method (SCM) based on reproducing kernel approximation", Comput. Methods Appl. Mech. Engrg., 371, Article ID 113303 (2020).

13. Wang, L., Wang, Z., and Qian, Z. "A meshfree method for inverse wave propagation using collocation and radial basis functions", Comput. Methods Appl. Mech. Engrg., 322(1), pp. 311-350 (2017).

14. Izadi, M. "A comparative study of two Legendrecollocation schemes applied to fractional logistic equation", Int. J. Appl. Comput. Math., 6(3), Article ID $71(2020)$.

15. Izadi, M. and Afshar, M. "Solving the Basset equation via Chebyshev collocation and LDG methods", $J$. Math. Model., 9(1), pp. 61-79 (2021).

16. Izadi, M. "Comparison of various fractional basis functions for solving fractional-order logistic population model", Facta Univ. Ser. Math. Inform., 35(4), pp. 1181-1198 (2020).

17. Izadi, M. and Srivastava, H.M. "Numerical approximations to the nonlinear fractional-order Logistic population model with fractional-order Bessel and Legendre bases", Chaos Solitons Fract., 145, pp. 1-11, Article ID 110779 (2021).

18. Krall, H.L. and Frink, O. "A new class of orthogonal polynomials: The Bessel polynomials", Trans. Amer. Math. Soc., 65, pp. 100-115 (1949).

19. Izadi, M. and Cattani, C. "Generalized Bessel polynomial for multi-order fractional differential equations", Symmetry, 12(8), Article ID 1260 (2020).

20. Izadi, M. "Numerical approximation of Hunter-Saxton equation by an efficient accurate approach on long time domains", U.P.B. Sci. Bull. Series A, 83(1), pp. 291300 (2021).

21. Izadi, M. and Cattani, C. "Solution of nonlocal fractional-order boundary value problems by an effective accurate approximation method", Appl. Ana. Optim., 5(1), pp. 29-44 (2021).

22. Izadi, M. and Srivastava, H.M. "An efficient approximation technique applied to a non-linear Lane-Emden pantograph delay differential model", Appl. Math. Comput., 401, pp. 1-11, Article ID 126123 (2021).

23. Izadi, M., Seifaddini, M., and Afshar, M. "Approximate solutions of a SIR epidemiological model of computer viruses", Adv. Stud. Euro-Tbilisi Math. J., 14(4), pp. 203-219 (2021).

24. Arbabi, S., Nazari A., and Darvishi, M.T. "A semianalytical solution of foam drainage equation by Haar wavelets method", Optik, 127, pp. 54-43 (2016).

25. Khan, M. and Gondal, M.A. "A new analytical solution of foam drainage equation by Laplace decomposition method", J. Adv. Res. Differ. Equ., 2, pp. 53-64 (2010).

\section{Biography}

Mohammad Izadi received his $\mathrm{PhD}$ degree from Leipzig University (2008-2012) in the group of "Scientific Computing" at Max-Planck Institute for Mathematics in the Sciences, Leipzig, Germany. Since 2013, he has served as an Assistant Professor at the Department of Applied Mathematics at Shahid Bahonar University of Kerman and since March 2021 is an Associated Professor. His interest areas include numerical analysis, numerical methods for (fractional) ordinary and partial differential equations, and spectral methods. 